\title{
The Establishment of Comprehensive Box Loading Plan
}

\author{
Yishuang $\mathrm{Hu}$ \\ School of electric power engineering, North China Electric Power University, Baoding 071000, China \\ 273415335@qq.com
}

Keywords: Integer programming, nonlinear programming, centroid equation.

\begin{abstract}
My model solves the problem of pallet loading problem with the limition of weight bearing. Model one calculate the number for two kinds of boxes putting in the pallet without overlap, which can make the best use of the pallet. Using Integer programming, I choose an arbitrary rectangular area in the lower left corner of the pallet, where I place box X in it. Secondly, I divide the left area into two part B and C in the way of transverse or vertical cutting, where I put box $\mathrm{Y}$ in these two parts. Set the maximum residual using area as constraint, the minimum excess stock as the objective function. Model two consider the uniformity stress of pallet which influence the position of each box. Let the distance between equivalent center of gravity of boxes and center of gravity of pallet stands for the level of uniformity stress. As different box has different weight which makes impact on the uniformity stress, so I define modified parameter A to correct our equation. With the result gotten in model one, I can calculate the gravity position for each boxes using nonlinear programming.
\end{abstract}

\section{Introduction}

The tray has become one of the important symbol to measure the country's level of efficiency, study how to place the largest number of boxes on the pallet, which is the pallet loading problem has the greatest significance in reducing the cost of logistics and transport, improving the efficiency.

I establish a universal model to solve the problem of pallet loading problem with the limition of weight bearing.

\section{Assumption}

1. Assume all the boxes lie within the pallet.

2. Assume boxes can be treated as rectangular when projecting which means there is no overlap.

3. Assume the pallet can place enough boxes if necessary.

\section{Parameter}

\begin{tabular}{c|c|c}
\hline Parameter & Definition & [Unit] \\
\hline$x_{i} y_{i}$ & The coordinate of gravity point for each box. & $\mathrm{mm}$ \\
\hline$a_{i}, b_{i,} h_{i}$ & Length,width,height for each box & $\mathrm{mm}$ \\
\hline sum $_{i}$ & The total number for the ith box & $\mathrm{mm}$ \\
\hline$L, W$ & Length,width for pallet & $\mathrm{mm}$
\end{tabular}

\section{Model one. Number solving model: find the number for each box.}

As there are two kind of putting ways, two kinds of cutting ways, two kinds of boxes. So totally speaking, there are eight circumstances that should be discussed.

1. Set the sides of pallet as X and Y coordinate in models. Suppose the length of pallet is $1200 \mathrm{~mm}$, and width is $1000 \mathrm{~mm}$. 
Table 1: the parameters of boxes in two models

\begin{tabular}{c|c|c|c|c}
\hline types & Weight $(\mathrm{kg})$ & $\mathrm{A}(\mathrm{mm})$ & $\mathrm{B}(\mathrm{mm})$ & $\mathrm{H}(\mathrm{mm})$ \\
\hline $\mathrm{X}$ & 10.132 & 370 & 250 & 135 \\
\hline $\mathrm{Y}$ & 3.289 & 330 & 150 & 205
\end{tabular}

2. Choose the lower left corner, putting box $\mathrm{X}$ which has two putting ways. Emphasis that, all the boxes should be put in the same direction.

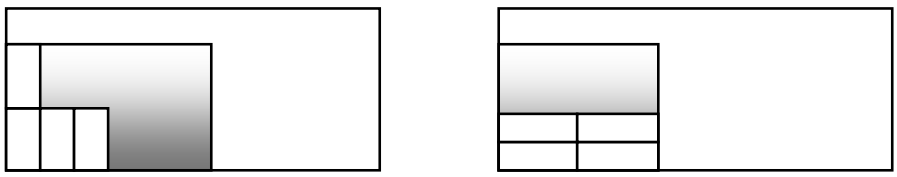

Figure 1: two putting ways in the chosen area

The restraint equation is: to make sure no boxes is outside the chosen area.

Note that: $\mathrm{M}$ is the number of $\mathrm{X}$ in the length of pallet, where $\mathrm{N}$ is the number of $\mathrm{X}$ in the width of pallet.

$$
\left\{\begin{array}{l}
1 \leq M \leq \frac{1200}{a_{1}} \\
1 \leq N \leq \frac{1000}{b_{1}}
\end{array}\right.
$$

3. There are two ways of cutting in the left area where we put box Y in it.
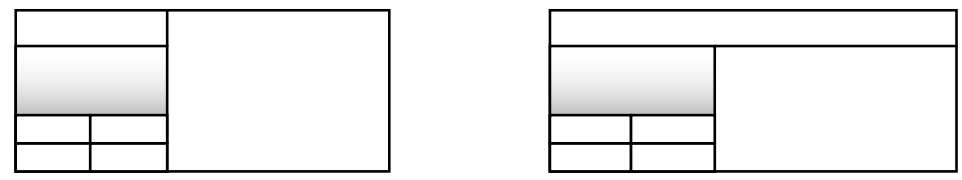

Figure 2: two cutting ways in the left area

4. Define $\mathrm{S}_{\mathrm{B}}, \mathrm{S}_{\mathrm{C}}$. which is the maximum using area in area $\mathrm{B}$ and $\mathrm{C}$, emphasis that, box $\mathrm{Y}$ in these area is putting in the same direction

$$
\begin{aligned}
& S_{B}=\max \left(\left[1200 / a_{5}\right] \times\left[\left(1000-N b_{1}\right) / b_{5}\right] a_{5} b_{5},\left[1200 / b_{5}\right] \times\left[\left(1000-N b_{1}\right) / a_{5}\right] a_{5} b_{5}\right) \\
& S_{C}=\max \left(\left[\left(1200-M a_{1}\right) / a_{5}\right] \times\left[N b_{1} / b_{5}\right] a_{5} b_{5},\left[\left(1200-M a_{1}\right) / b_{5}\right] \times\left[N b_{1} / a_{5}\right] a_{5} b_{5}\right)
\end{aligned}
$$

5. Take the cutting way as cross cutting and putting as example, its Integer programming is list below.

$$
\begin{aligned}
& g_{1}=\min \left(1200 \times 1000-N M a_{1} b_{1}-S_{B}-S_{C}\right) \\
& \text { s.t }\left\{\begin{array}{l}
1 \leq M \leq \frac{1200}{a_{1}} \\
1 \leq N \leq \frac{1000}{b_{1}} \\
S_{B}=\max \left(\left[1200 / a_{5}\right] \times\left[\left(1000-N b_{1}\right) / b_{5}\right] a_{5} b_{5},\left[1200 / b_{5}\right] \times\left[\left(1000-M b_{1}\right) / a_{5}\right] a_{5} b_{5}\right) \\
S_{C}=\max \left(\left[\left(1200-M a_{1}\right) / a_{5}\right] \times\left[N b_{1} / b_{5}\right] a_{5} b_{5},\left[\left(1200-M a_{1}\right) / b_{5}\right] \times\left[N b_{1} / a_{5}\right] a_{5} b_{5}\right)
\end{array}\right.
\end{aligned}
$$

6. swap the type of boxes in each area, and changing its cutting, putting ways, after all, we can get eight Integer programming equations, using MATLAB we can obtain its eight integer solving results ,then compared these eight answers, we can achieve the best number of two boxes which make the best use of this pallet.

Conclusion in model one: we need box X 1, box Y 19. 


\section{Model two. Position map model: find position for each box that ensures pallet bear the stress evenly.}

Consider the uniformity stress of pallet which influence the position of each box. Let the distance between equivalent center of gravity of boxes and center of gravity of pallet stand for the level of uniformity stress. As the different box has different Iight which makes impact on the uniformity stress, so I define modified parameter A to correct our equation.with the result gotten in model one, I can calculate the gravity position for each boxes using nonlinear programming.

1. Define modified parameter A to ensure the gravity distance equation can represent the uniformly stress.

$$
\frac{m_{k}}{V_{k}}=A \frac{m_{l}}{V_{l}}
$$

So the modified objective equation which is the distance between equivalent center of gravity of boxes and center of gravity of pallet

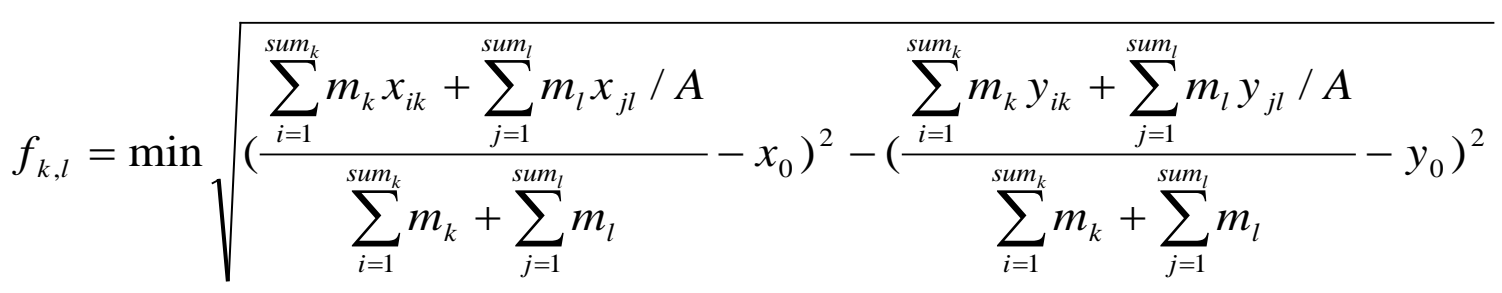

2. The nonlinear programming equation is;

Note that: the constraint conditions refer to the situation that no boxes is outside the pallet, no boxes is overlap.

$\left(x_{i k} y_{i k}\right)$ is the coordinate of ith box for box $\mathrm{K}$

$\left(\begin{array}{ll}x_{j l} & y_{j l}\end{array}\right)$ is the coordinate of jth box for box $\mathrm{L}$

$$
\text { s.t. }\left\{\begin{array}{l}
\left(x_{i k}-x_{j k}\right)^{2}+\left(y_{i k}-y_{j k}\right)^{2} \geq b_{k}^{2} \\
\left(x_{i l}-x_{j l}\right)^{2}+\left(y_{i l}-y_{j l}\right)^{2} \geq b_{l}^{2} \\
\left(x_{i k}-x_{j l}\right)^{2}+\left(y_{i k}-y_{j l}\right)^{2} \geq\left(\frac{1}{2} b_{k}+\frac{1}{2} b_{l}\right)^{2} \\
\frac{1}{2} a_{k} \leq x_{i k}, x_{j l} \leq 1200-\frac{1}{2} a_{k} \\
\frac{1}{2} a_{k} \leq y_{i k}, y_{j l} \leq 1000-\frac{1}{2} a_{k} \\
\frac{1}{2} a_{l} \leq x_{i k}, x_{j l} \leq 1200-\frac{1}{2} a_{l} \\
\frac{1}{2} a_{l} \leq y_{i k}, y_{j l} \leq 1000-\frac{1}{2} a_{l}
\end{array}\right.
$$

3. Submit the necessary information in the equation with box $\mathrm{X}$ and box $\mathrm{Y}$ to get the final position for each box, which can ensure two factor: First, the number of boxes can take upmost area of pallet. Second, the position of boxes guarantee the evenly stress the pallet bear.

Conclusion in model one: see table two, it shows the directly coordinate of boxes and the value of the distance defined in model two is $3.7788 \mathrm{e}-06 \mathrm{~mm}$, which means the pallet bear the same stress around every part. Based on the number of boxes calculated in model one, box X is 1 and box Y is 19 . 
And the coordinate calculated in model two, we can easily map the placement direction for each box which is the result of Pallet loading problem.

Table 2: the directly coordinate of boxes (partly 5 among 20)

\begin{tabular}{c|c}
\hline $\mathrm{X} * 1.0 \mathrm{e}+03 \mathrm{~mm}$ & $\mathrm{Y} * 1.0 \mathrm{e}+03 \mathrm{~mm}$ \\
\hline$(\mathrm{X}) 0.6907$ & $(\mathrm{X}) 0.8150$ \\
\hline$(\mathrm{Y}) 0.4501$ & $(\mathrm{Y}) 0.3839$ \\
\hline$(\mathrm{Y}) 0.3810$ & $(\mathrm{Y}) 0.6644$ \\
\hline$(\mathrm{Y}) 0.9835$ & $(\mathrm{Y}) 0.5090$ \\
\hline$(\mathrm{Y}) 0.3165$ & $(\mathrm{Y}) 0.8012$
\end{tabular}

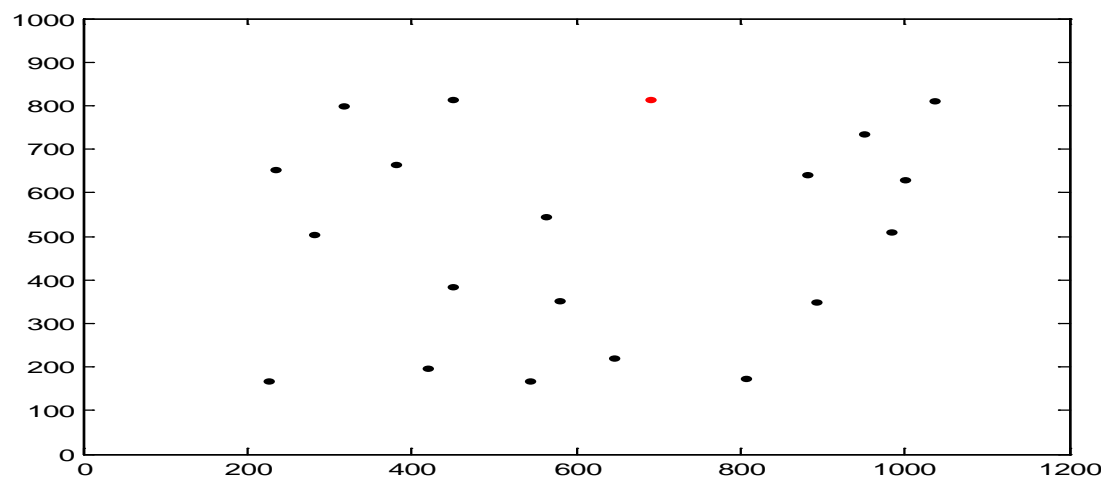

Figure 3: the map of position

\section{Future application}

My model solve the layout of two boxes considering the usage and the bearing stress, but our model can be promoted in the three or more than three boxes layout problem, which is much more suitable for the reality. The mainly method is integer programming in model one to calculate the number of boxes that cover the pallet as maximum as it can be. The nonlinear programmer in model two to get the gravity position of each box ensuring the stress evenly distributed in the pallet with modified parameter A.

\section{Reference}

[1] Yong Li, The approximate algorithm in the application of layout optimization, Huazhong University of science and technology, 2005. 